\title{
El ganglio centinela en el melanoma: nuestra experiencia
}

\section{The sentinel Iymph node biopsy for melanoma: our experience}

\author{
Martí Carrera, E.*, Díaz Gutiérrez, A.J .**, Martín Díaz, M.*, González Vecino, M.L.*, \\ Sánchez Crespo, 0.*, Lagarón Comba, E.J .***, García Martínez, A.*****
}

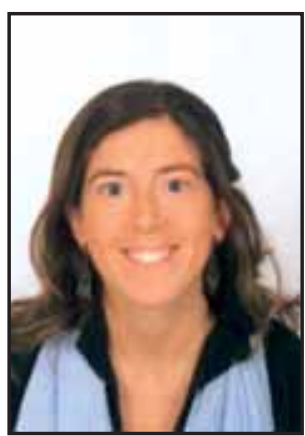

Martí Carrera, E.

\section{Resumen}

El ganglio centinela ha sido recientemente aceptado como uno de los factores pronósticos más importantes en el melanoma maligno según la AJCC (American Joint Committee on Cancer), a pesar de que su uso sistemático no está aún extendido a todas la unidades de melanoma y su empleo no está exento de controversias. Entre otros aspectos, se discute si la realización de la ampliación de márgenes debe ser realizada en el mismo tiempo quirúrgico que el ganglio centinela para la eficacia del mismo. Se realiza un estudio retrospectivo sobre 78 pacientes intervenidos de ganglio centinela por melanoma entre Mayo 99 y Enero 04, todos ellos remitidos por Dermatología con diagnóstico de melanoma maligno $>1 \mathrm{~mm}$ de espesor de Breslow y adenopatías no palpables (estadio I y II de la AJCC). De ellos, 42 son mujeres y 36 varones, con una edad media de 53.5 años. El espesor de Breslow medio es de $2.78 \mathrm{~mm}$. La ampliación de márgenes se hizo en un tiempo quirúrgico anterior por parte de Dermatología en 59 casos (76.62\%), mientras que en 18 se realizó en el mismo tiempo que el ganglio centinela. La demora media entre la ampliación de márgenes y la realización del ganglio centinela fue de 5.87 meses. En el 19.23\% (15 pacientes) no se observó captación gamagráfica, por lo que el estudio del ganglio centinela no pudo llevarse a cabo. De ellos, en 12, la ampliación de márgenes había sido anterior al tiempo del ganglio centinela, con una demora media de 5.06meses.E1 50\% (39) de los ganglios enviados a Anatomía Patológica fueron negativos para células malignas. $\mathrm{El} 25.74 \%$ (20pacientes) fueron positivos. El seguimiento medio es de 23.12 meses; 18 pacientes presentan en la actualidad metástasis o han fallecido; 47 casos $(60 \%)$ se encuentran libres de tumor. Según la experiencia recogida en nuestro Servicio durante este tiempo, se corrobora que el ganglio centinela es una técnica sencilla, de baja morbilidad, con una importante función de estadiaje para el tratamiento del melanoma maligno. No se observa ninguna diferencia entre el grupo de casos con ampliación de márgenes en el tiempo del ganglio centinela y aquellos con ampliación previa.

Palabras clave Melanoma; Ganglio centinela

Código numérico 1720
The sentinel lymph node biopsy (SLNB) has recently been accepted as one of the most important prognostic factors of the cutaneous primary melanoma by the AJCC (American Joint Committee on Cancer), even its overall use is still not extended to all the melanoma units and there are many controversies about its use. Among other aspects, there is no consensus about if the previous wide local excision can alter the results of the biopsy. This is a retrospective study done over 78 consecutive cases of SLNB for melanoma between May'99 and Jan'04. All of them were remitted from Dermatology with the diagnose of melanoma, tumor thickness $>1.0 \mathrm{~mm}$ and no clinical nodes (AJCC est. I and II). Out of the 78 cases, 42 were women and 36 men, with an average age of 53.5. The average Breslow thickness was of $2.78 \mathrm{~mm}$. The wide local excision(WLE) was done by Dermatology at a previous time in 59 cases (76.62\%), while 18 patients had the WLE at the time of the SLNB. The delay between the WLE and the SLNB was of 5.87 months. In the $19.23 \%$ (15patients) the SLNB was not feasible because there was not found colloid captation by any node; 12 of them had de WLE previous to the SLNB, with a delay of 5.06 months; $50 \%$ (39) of the nodes were negative for melanoma cells; $25.74 \%$ (20patients) were positive. The follow up time was of 23.12 months; 18 patients presented metastasis or died, while 47 cases $(60 \%)$ are actually free of disease. We corroborate that the SLNB is a low morbility technique with an important staging function for the primary cutaneous melanoma. We don't find any difference in the survival of patients with previous WLE compared with those whom the WLE was done concurrently.
Key words Melanoma; Sentinel limph node

Código numérico 1720

Médico Residente Cirugía Plástica, Hospital Doce de Octubre, Madrid (España). Especialista en Cirugía Plástica. Práctica Privada. 
Introducción

Desde que Morton describió en 1992 el ganglio centinela para el despistaje de metástasis ganglionares de melanoma (1), su uso se ha generalizado en muchas unidades de melanoma, de modo que actualmente se considera, junto al espesor de Breslow y la ulceración del tumor, uno de los factores pronósticos más importantes del melanoma maligno (MM) (2-5, 1) La técnica está basada en la hipótesis (ahora bien establecida), de que las metástasis linfáticas del MM siguen una progresión ordenada a través de los canales linfáticos aferentes hacia el ganglio centinela antes de repartirse hacia otros ganglios regionales. Es un método específico y mínimamente invasivo para identificar a los pacientes con metástasis ganglionares ocultas, y evitar, de esta forma, la linfadenectomía electiva a un gran número de pacientes sin afectación ganglionar que no van a beneficiarse de la misma. En manos experimentadas, este procedimiento diagnóstico ofrece una información comparable a la linfadenectomía con una morbilidad considerablemente menor.

Sin embargo, su uso no está exento de controversias y aún están en debate muchos factores sobre su realización y manejo. Se calcula que aproximadamente un $3 \%$ de los ganglios centinela negativos resultan ser falsos negativos (6). Se han propuesto muchas explicaciones para estos casos. Entre ellas se propone que la ampliación de márgenes en un tiempo previo a la biopsia del ganglio supone una irrupción de los canales linfáticos y por lo tanto una alteración de las líneas de Sappey que artefacta el flujo hacia la cadena ganglionar y dificulta la localización del ganglio centinela (7-10). Se ha establecido que la realización del ganglio centinela debe llevarse a cabo después de la biopsia inicial y antes de la ampliación de márgenes, ya que el drenaje linfático puede estar profundamente modificado con los procedimientos quirúrgicos

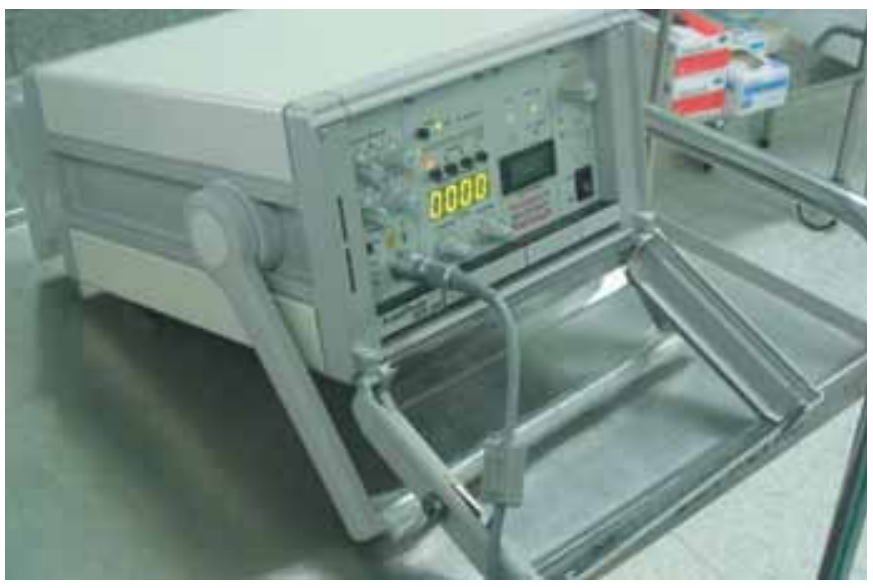

Foto 1: Gammacámara previos. Recientemente han aparecido nuevos artículos desechando esta teoría y demostrando que la ampliación de márgenes en un tiempo anterior al ganglio centinela no es una contraindicación para la realización del mismo, siempre que la ampliación sea menor de $2 \mathrm{~cm}$ y no se realice cobertura con colgajos (11-14).

El propósito de esta revisión es evaluar los resultados obtenidos en nuestro Servicio de Cirugía Plástica del Hospital Doce de Octubre de Madrid (España) desde que se lleva a cabo la realización sistemática del ganglio centinela (Mayo del 99) y valorar si la ampliación de márgenes previa a la realización del ganglio centinela artefacta el resultado del mismo y/o ensombrece el pronóstico.

\section{Material y Método}

Se realiza un estudio retrospectivo de todos aquellos pacientes que fueron remitidos al Servicio de Cirugía Plástica del hospital Doce de Octubre de Madrid para la realización de ganglio centinela entre Mayo del 99 y Enero de 04. Se trata de 78 pacientes remitidos por el servicio de Dermatología con diagnóstico de melanoma maligno estadio clínico I (Estadio I y II de la AJCC, ganglios palpables (-)/ metástasis a distancia (-), y $>1 \mathrm{~mm}$ de Breslow). Se descartan aquellos pacientes con $\mathrm{MM}<1 \mathrm{~mm}$ de profundidad porque se ha demostrado que no se benefician de la linfadenectomía electiva (15), excepto en aquellos casos con otros factores de riesgo de mal pronóstico (ulceración o signos anatomopatológicos de regresión espontánea) que sí se incluyen en el estudio.

En general la ampliación de márgenes se realiza por parte del servicio de Dermatología siguiendo los siguientes criterios:

$M M<1 \mathrm{~mm}$ de espesor: $1 \mathrm{~cm}$ de margen.

MM>1mm: $2 \mathrm{~cm}$ de margen en función de su localización.

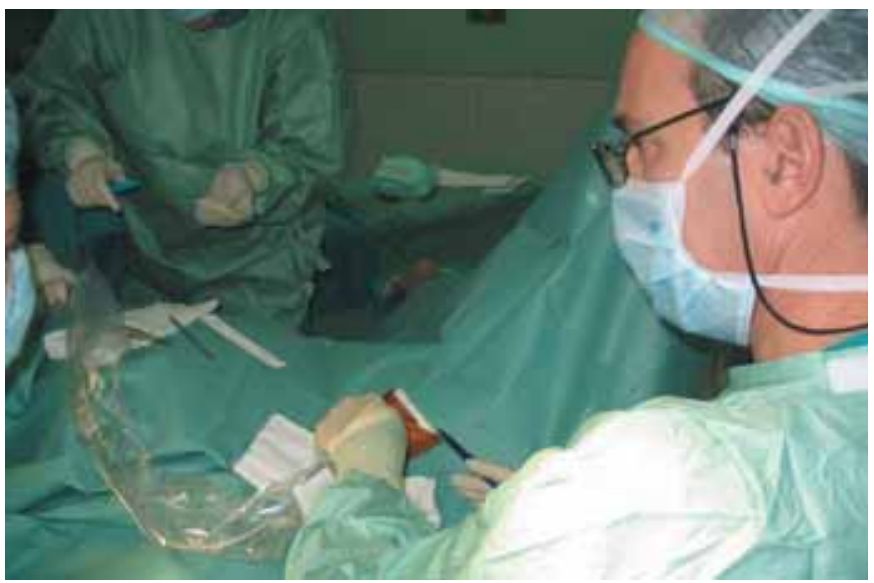

Foto 2: Comprobación de puntos de referencia. 


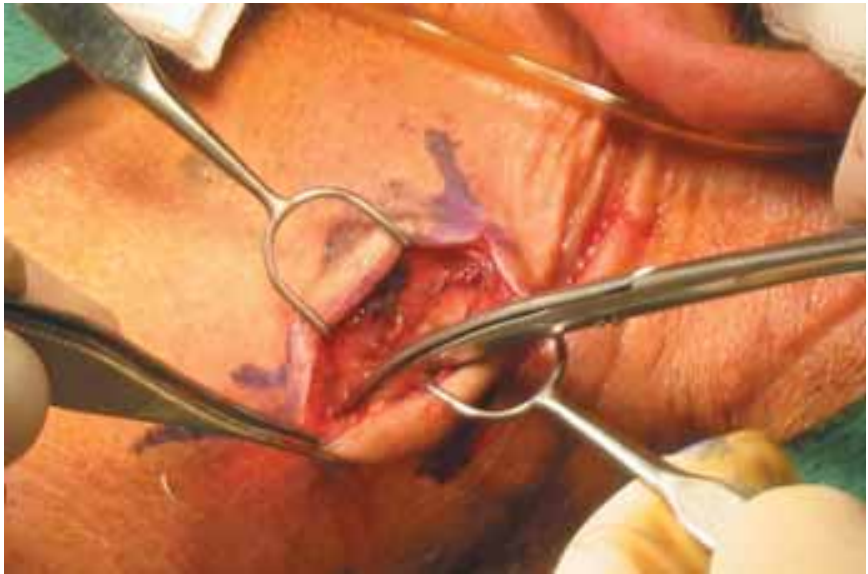

Foto 3. Disección

MM subungueal: amputación desde la interfalángica distal (IFD).

La ampliación se realiza bien por cierre directo del defecto, bien por cicatrización por segunda intención. En ningún caso se realizan colgajos para la cobertura.

Se remiten a Cirugía Plástica aquellos pacientes cuya ampliación de márgenes requiera injerto o plastia; en tal caso, la ampliación de márgenes y la detección del ganglio centinela se realizan en el mismo acto quirúrgico.

El día de la cirugía el paciente es trasladado a Medicina Nuclear para la inyección intradérmica de radioisótopo, Tecnecio 99- sulfuro de antimonio, en los cuatro puntos cardinales de la lesión, y se realiza la linfoscintigrafía dinámica y estática, observando aquellos ganglios a los que primero drena el trazador, para marcarlos como "puntos calientes".

En quirófano se realiza la detección del ganglio centinela con anestesia local, en caso de que no sea precisa la ampliación de márgenes o de que no haya contraindicación, con la ayuda de una sonda de detección gammagráfica, una gammacámara y el especialista en Medicina Nuclear (Fig. 1-6). Aunque hay autores que emplean colorantes asociados al radioisótopo para aumentar la sensibilidad de la técnica, la

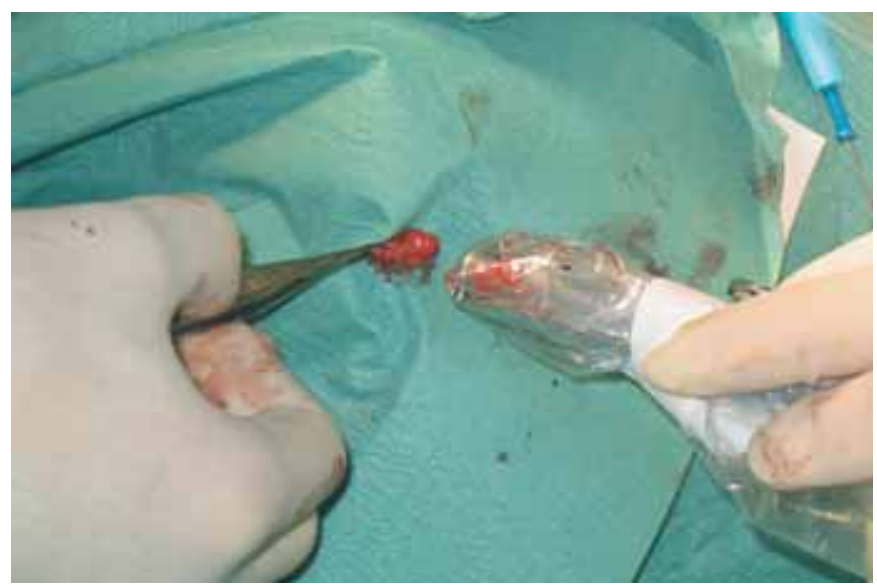

Foto 5: Comprobación del ganglio.

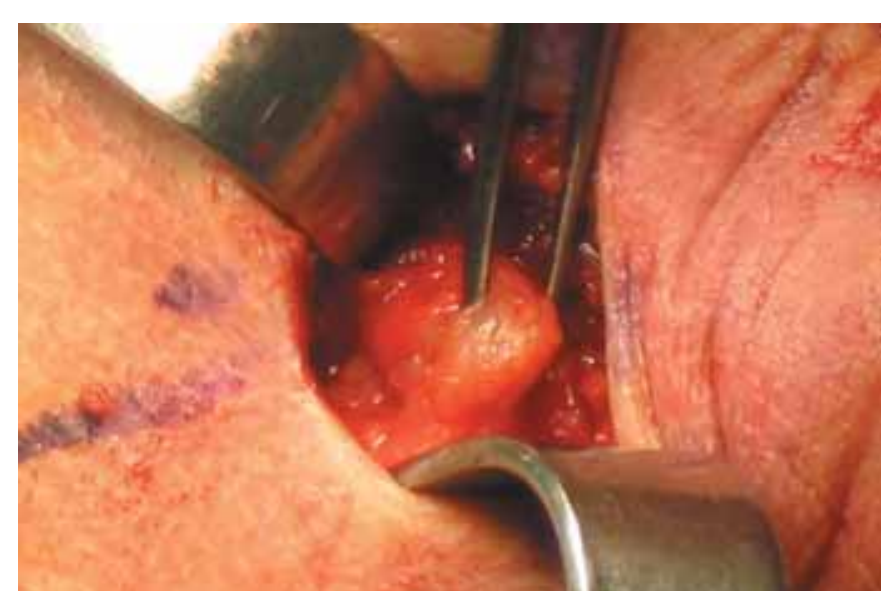

Foto 4: Localización del ganglio centinela.

gammagrafía sola tiene una tasa de éxito del $98 \%$ en manos expertas y el uso de tinciones se está abandonando (8). La pieza se envía en fresco para su estudio en Anatomía Patológica.

El estudio histopatológico se realiza mediante tinción con hematoxilina-eosina en cortes seriados, e inmunohistoquímica. Sólo las secciones congeladas rutinarias no se recomiendan por el alto índice de falsos negativos, y la asociación de ambas técnicas parece demostrar gran mejoría en la detección de metástasis microscópicas (16-18). Las tinciones con hematoxilina-eosina son capaces de detectar una célula maligna de cada 10000 células, y detección que asciende a una de cada 100000 células con la inmunohistoquímica. Esta consiste en la tinción de componentes específicos de los melanocitos (S-100, de los filamentos intermedios del citoplasma, HMB-45 y MelanA de los melanosomas). Durante un tiempo se recomendó la realización de PCR-transcriptasa inversa para la tirosinasa, pero actualmente esta técnica se ha desechado por ser excesivamente sensible (19).

La linfadenectomía se realiza en caso de que el ganglio centinela sea positivo y que no esté contraindicada (por importante deterioro del estado general del paciente o por rechazo por parte del mismo).

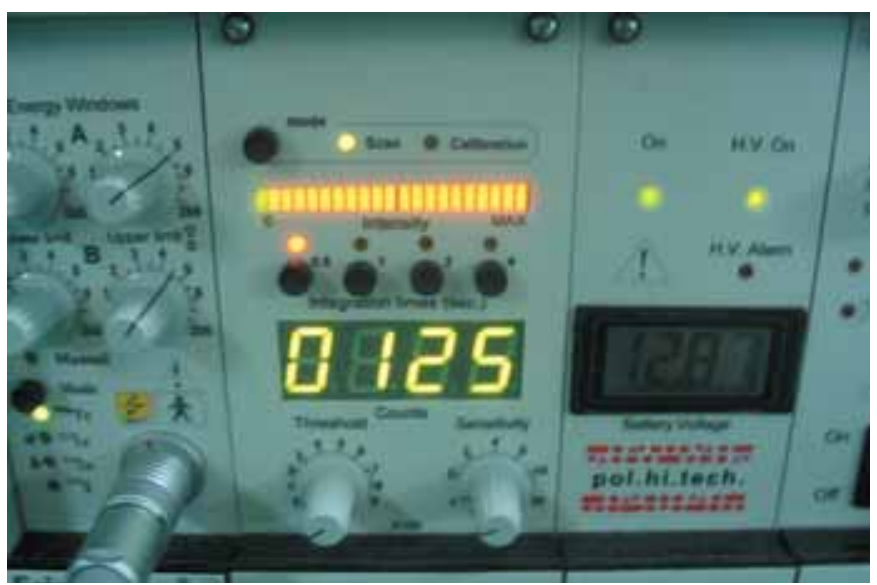

Foto 6: Contaje isotópico por gammacámara. 
Gráfico 1.

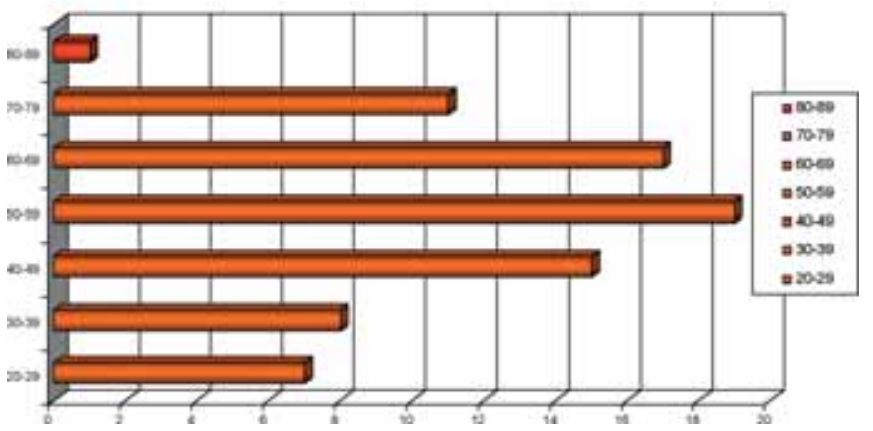

Edad de los pacientes estudiados

Gráfico 2.

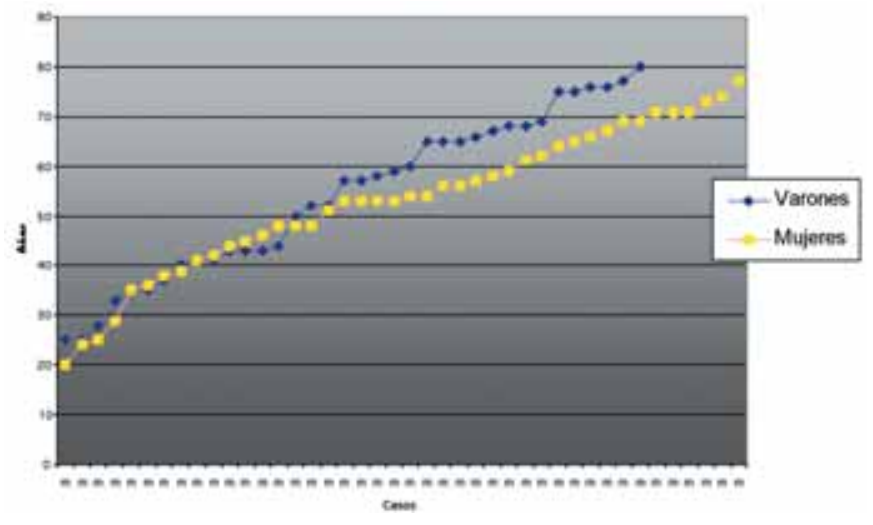

Distribución de edad según sexos

Gráfico 3.

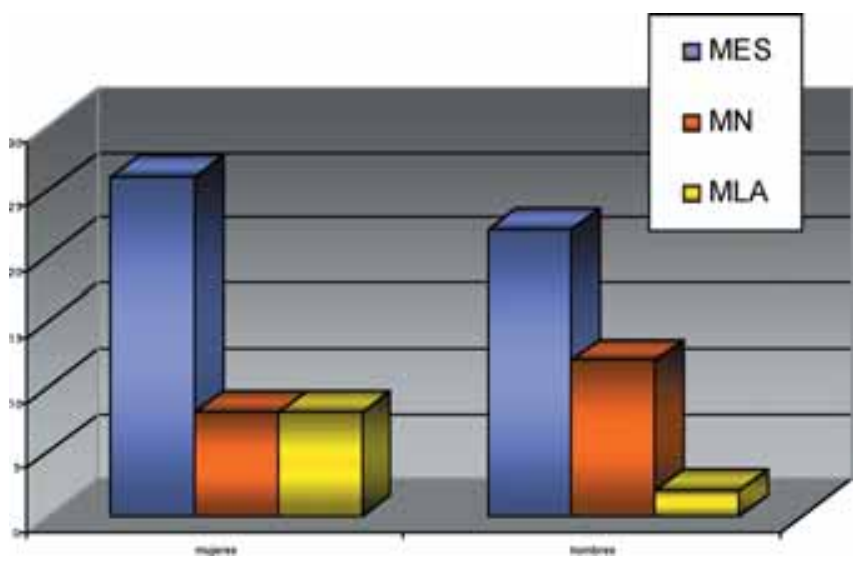

Tipo de melanoma según sexos

Gráfico 4.

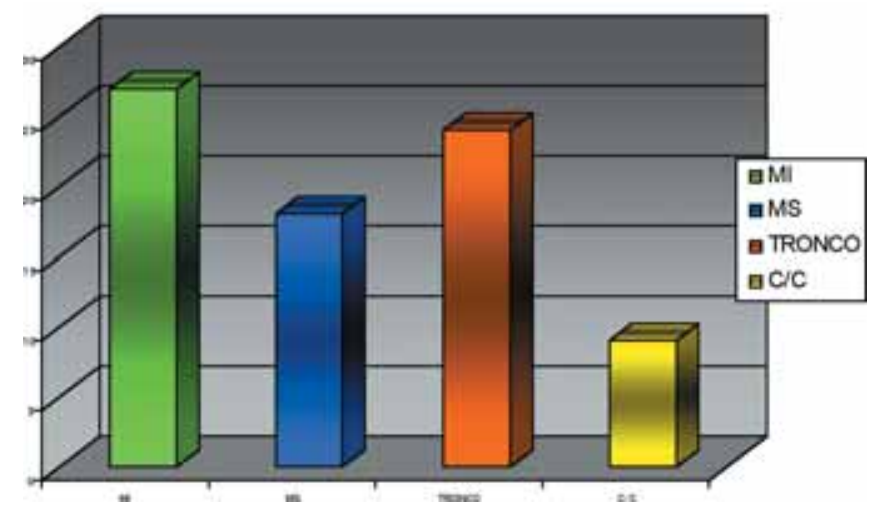

Localización tumoral
Tras la linfadenectomía el paciente es nuevamente remitido a Dermatología para valorar tratamiento con interferón.

En caso de ganclio centinela negativo (G.C. (-)), el paciente se remite a Dermatología para valorar el tratamiento con vacuna de gangliósidos $(\mathrm{MM}>1.5 \mathrm{~mm}$ con G.C(-)) y continuar el seguimiento.

Los pacientes son remitidos a Oncología en caso de aparición de metástasis a distancia, para valorar tratamiento quimioterápico o radioterapia.

\section{Resultados}

De los 78 pacientes que fueron sometidos a estudio de ganglio centinela en el periodo comprendido entre Mayo de 1999 y Enero de 2004, 42 eran mujeres y 36 varones, con una edad media de 53,5 años (rango entre 20 y 80 años). (Gráfico 1). No existían diferencias significativas en la distribución de la edad según sexos (Gráfico 2).

El tipo histológico más frecuente fue el Melanoma de Extensión Superficial (MES), en 48 pacientes (61.5\%), seguido por el Melanoma Nodular (MN) en 20 casos $(25.64 \%$ casos $)$ y por el Melanoma Lentiginoso Acral (MLA) en 10 pacientes $(7,8 \%)$.

No se diagnosticó ningún léntigo maligno melanoma. La distribución por sexos fue similar, a excepción del melanoma lentiginoso acral que en 8 de 10 casos se presentó en mujeres, sin embargo, la diferencia no es estadísticamente significativa (Gráfico 3).

La localización tumoral más frecuente fue en el miembro inferior (34.6\% casos), seguida del tronco $(30.77 \%)$, el miembro superior $(23.08 \%)$ y, por último, cabeza y cuello (11.53\%) (Gráfico 4).

Sin embargo, al valorar la localización del tumor inicial según el sexo, encontramos que 22 de los 36 pacientes varones de la serie $(61.11 \%)$ tenían originariamente el melanoma en región de mal pronóstico (tronco-cara/cuello), mientras que el $73.81 \%$ de las mujeres presentaban la lesión en miembro superior o inferior, mediante un análisis de probabilidades $\chi^{2}$ la $\mathrm{p}=0.0019$ ( Gráfico 5).

El espesor de Breslow medio fue de $2.78 \mathrm{~mm}$, con un rango entre 1.0 y $10.3 \mathrm{~mm}$. (Gráfico 6,7 ).

El espesor medio en mujeres fue de $2.28 \mathrm{~mm}$, mientras que el espesor medio en varones fue de $3.40 \mathrm{~mm}$. En el análisis de probabilidades $\chi^{2} \mathrm{p}=0.0054$. (Gráfico 8).

La ampliación de márgenes se realizó por el Servicio de Dermatología en un tiempo quirúrgico previo al ganglio centinela en 59 pacientes (76.62\%). El tiempo de demora medio entre la ampliación de márgenes y la detección del ganglio centinela fue de 5.87meses. En 18 casos (23.38\%) se realizó en el mismo tiempo quirúrgico por parte de Cirugía Plástica. 
Gráfico 5.

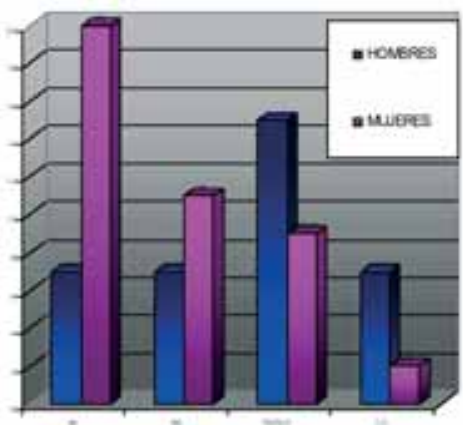

Localización según sexo

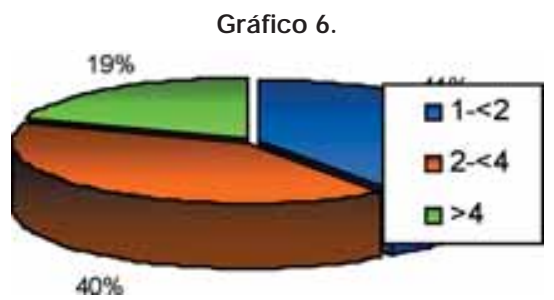

Espesor de Breslow

Gráfico 7.

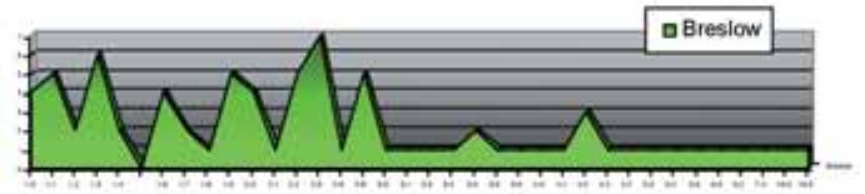

Espesor de Breslow

Gráfico 8.

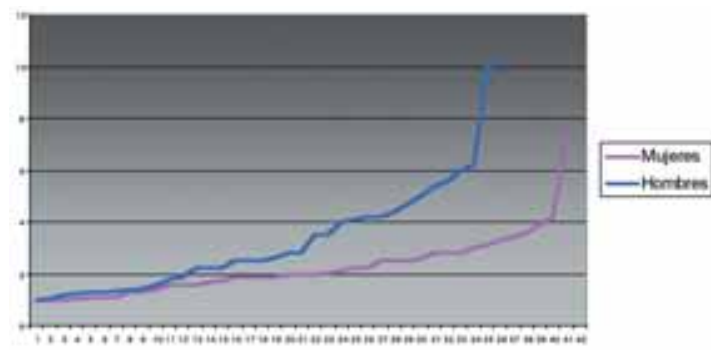

Espesor medio según sexos

Gráfico 9.

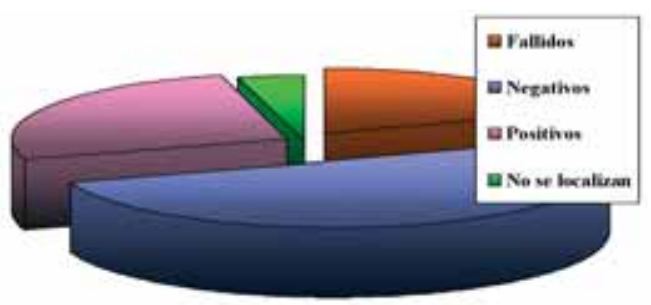

Estudio ganglio centinela

Gráfico 10.

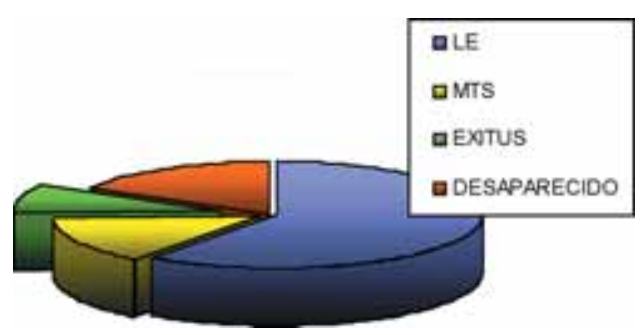

El ganglio centinela fue positivo en 20 casos $(25.74 \%)$ y negativo en 39 casos (50\%). En 15 pacientes $(19.23 \%)$ la detección del ganglio centinela resultó fallida por parte del Servicio de Medicina Nuclear, al no conseguir captar en la linfoscintigrafía ningún punto caliente tras la inyección de radioisótopo. En tres casos no se logró la detección del ganglio centinela por parte del cirujano en el quirófano, por dificultad de localización del mismo (Gráfico 9).

En 19 de los 20 pacientes con ganglio centinela positivo se realizó linfadenectomía programada. En un caso se desestimó la cirugía dada que la situación clínica del paciente era muy precaria. La demora media entre la detección del ganglio centinela y la linfadenectomía fue de 30 días.

El seguimiento medio ha sido de 23.12 meses. Se ha perdido la evolución de 13 pacientes (16.67\%); 7 han fallecido $(8.97 \%)$; 11 han presentado durante el seguimiento metástasis, bien locales, ganglionares, o a distancia; 47 casos $(60.26 \%)$ se encuentran en la actualidad libres de tumor (Gráfico 10).

De los 39 pacientes con ganglio centinela negativo, 6 presentaron metástasis a lo largo del seguimiento (15.38\%) (Tabla I). El 35.0\% de los casos con ganglio centinela positivo desarrollaron metástasis en el mismo tiempo de seguimiento (Tabla II). Tres pacientes con G.C.(-) presentaron metástasis ganglionares en menos de un año de seguimiento, es decir, se trató de falsos negativos. El tiempo de demora medio entre la ampliación de márgenes y el ganglio centinela fue de 4.6 meses. En un caso la localización del tumor inicial fue el miembro superior, en otro caso el miembro inferior y en el tercero el tronco. El espesor de Breslow medio fue de $4.43 \mathrm{~mm}$.

Tabla I

$\begin{array}{lccc} & \text { MTS sí } & \text { MTS no } & \\ \text { G.C (-) } & 6 & 33 & \mathbf{1 5 . 3 8 \%} \\ \text { G.C. no (-) } & 12 & 27 & \mathbf{3 0 . 7 7 \%}\end{array}$

Tabla II

\begin{tabular}{lccr}
\multicolumn{3}{c}{ MTS sí } & MTS no \\
G.C (+) & 7 & 13 & $\mathbf{3 5 . 0 \%}$ \\
G.C. no (+) & 11 & 46 & $\mathbf{2 3 . 4 0 \%}$
\end{tabular}

La media del nivel de Breslow en milímetros para aquellos pacientes que se encuentran en la actualidad libres de tumor es de $2.43 \mathrm{~mm}$ (DE: 1.63 ), mientras que para los pacientes que han presentado metástasis o han fallecido es de 3.68.. (DE: 2.11). p>0.0076.

Dado que la demora entre la ampliación de márgenes y el ganglio centinela en el hospital es de varios meses, quisimos saber cuál era la relación entre la 
ampliación de márgenes previa y los ganglios centinela fallidos y entre la ampliación de márgenes y el pronóstico. La demora media entre los pacientes con ganglio centinela fallido fue de 5.06 meses (DE: 4.41). Entre los pacientes en que el ganglio centinela resultó negativo fue de 4.28 meses (DE:3.74). En aquellos con ganglio centinela positivo, la demora media fue de 4.72 meses (DE:7.72). Ambos grupos son homogéneos para sexo, edad, localización tumoral, tipo de melanoma y Breslow.

De los ganglios centinela fallidos, en el $80.0 \%$ la ampliación de márgenes se realizó en un tiempo anterior al ganglio, mientras que en el $75.80 \%$ de los no fallidos la ampliación de márgenes también se había presentado en un tiempo previo. Del mismo modo, en el $20.34 \%$ de los pacientes en que la ampliación de márgenes se realizó en un tiempo anterior el ganglio centinela resultó fallido; así como en el $16.6 \%$ de aquellos pacientes en que la ampliación se realizó en el mismo tiempo quirúrgico que el ganglio centinela (Tabla III).

Tabla III

$\begin{array}{cc}\text { DEMORA } & \text { DEMORA } \\ \text { SÍ } & \text { NO }\end{array}$

$\begin{array}{lrrr}\text { FALLIDOS } & 12 & 3 & \mathbf{8 0 . 0 \%} \\ \text { NO FALLIDOS } & 47 & 15 & \mathbf{7 5 . 8 0 \%}\end{array}$

$20.34 \% \quad 16.6 \%$

De los 45 pacientes a los que se les realizó la ampliación de márgenes en un tiempo quirúrgico anterior al ganglio centinela, el $33.3 \%$ dio positivo. El $35.7 \%$ de los 14 casos en que la ampliación y el ganglio se realizó en el mismo tiempo, también fue positivo. El 75.0\% de los ganglios negativos presentaba demora entre la ampliación de márgenes y el ganglio. El 76.9\% de los ganglios negativos también (Tabla IV).

Tabla IV

$\begin{array}{cc}\text { DEMORA } & \text { DEMORA } \\ \text { SÍ } & \text { NO }\end{array}$

$\begin{array}{llll}\text { G.C.(+) } & 15 & 5 & \mathbf{7 5 . 0 \%} \\ \text { G.C.(-) } & 30 & 9 & \mathbf{7 6 . 9 \%}\end{array}$

\section{$33.3 \% \quad 35.7 \%$}

El 77.7\% de los pacientes que presentaron metástasis a lo largo del seguimiento presentaban una amplia- ción de márgenes previa, con una media de 4.39 meses de demora (DE: 5.451) al igual que el $76.2 \%$ de los pacientes que no desarrolló metástasis. En este grupo, la media fue de 4.45 meses (DE: 3.664). La correlación entre ambas variables es p:0.96. De los pacientes a los que se les realizó la ampliación de márgenes en un tiempo quirúrgico previo, el $23.73 \%$ desarrollaron metástasis. El 22.22\% de los pacientes en que se realizó en el mismo tiempo quirúrgico desarrollaron metástasis. La correlación chi2: 0.8948 (Tabla V).

Tabla V

\begin{tabular}{lccc}
\hline & $\begin{array}{c}\text { DEMORA } \\
\text { Sí }\end{array}$ & $\begin{array}{c}\text { DEMORA } \\
\text { NO }\end{array}$ & \\
& & & \\
MTS (+) & 14 & 4 & $\mathbf{7 7 . 7 \%}$ \\
MTS (-) & 45 & 14 & $\mathbf{7 6 . 2 \%}$ \\
& & & \\
& $\mathbf{2 3 . 7 3 \%}$ & $\mathbf{2 2 . 2 2 \%}$ & \\
\hline
\end{tabular}

En el análisis de supervivencia entre ambos grupos (demora 1: ampliación de márgenes previa/ demora 0: no ampliación previa), la probabilidad de supervivencia en nuestro estudio a los dos años es del 0.9394 para el grupo demora:0. Para el grupo demora:1, la probabilidad de supervivencia a los dos años es de 0.8269. La comparación entre el análisis de supervivencia para ambos grupos se muestra en el gráfico 11.

\section{Discusión}

En la última versión del sistema de estadiaje de la AJCC se incluye el ganglio centinela como uno de los factores pronósticos más importante en el melanoma. (3). En nuestro hospital se lleva a cabo la realización sistemática del ganglio centinela desde Mayo de 1999 para todos aquellos melanomas estadio I y II de la AJCC.

El estudio incluye 78 pacientes con una edad media de 53 años. La predilección por sexos no es significativa, puesto que se trata de 42 mujeres y 36 varones. La distribución de la edad por sexos es muy similar para ambos grupos.

La localización tumoral en regiones de mal pronóstico es mucho más frecuente en hombre que en mujeres, observándose una diferencia estadísticamente significativa (p:0.0019).

El tipo histológico de melanoma más frecuente es el MES, seguido por el MN y por el MLA. No existe en nuestra casuística ningún léntigo maligno melanoma, probablemente debido al lento desarrollo del tumor y al diagnóstico precoz de las lesiones precursoras por parte del servicio de Dermatología. 


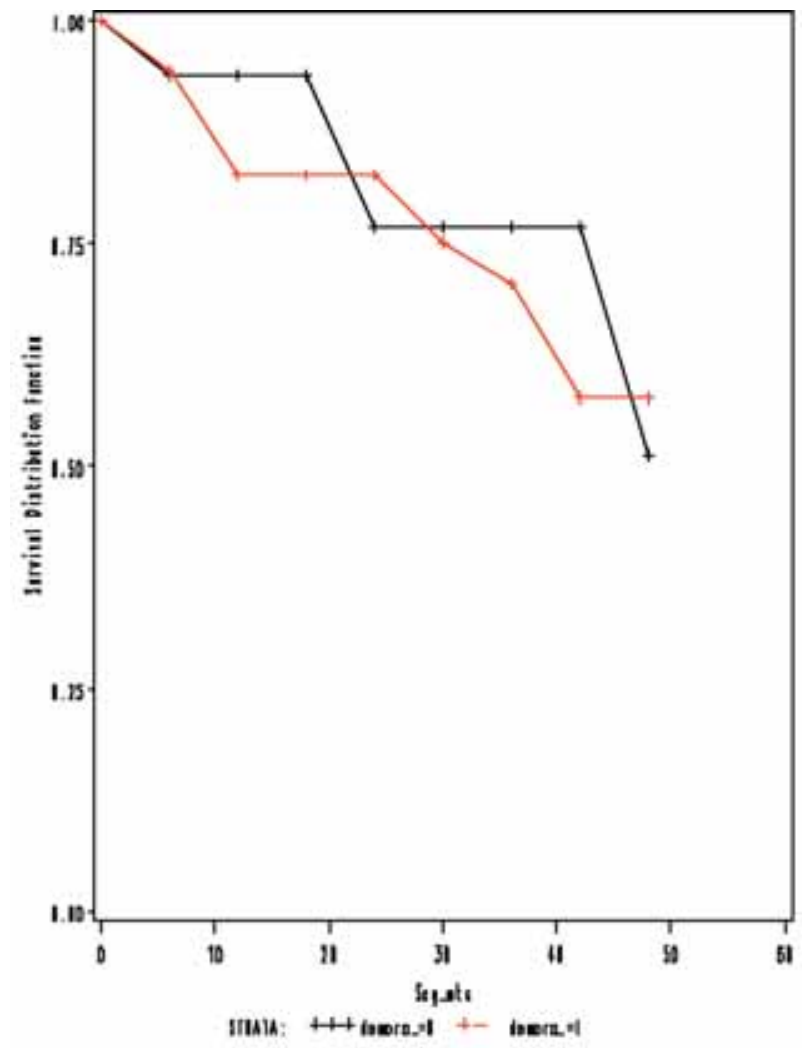

El espesor de Breslow medio fue de $2.78 \mathrm{~mm}$, con un rango entre 1.0 y $10.3 \mathrm{~mm}$. Existe una diferencia estadísticamente significativa (p:0.0054) entre el espesor de Breslow en mujeres $(2.28 \mathrm{~mm})$ en el momento del diagnóstico, frente al de los hombres $(3.40 \mathrm{~mm})$. Aunque este dato precisa un estudio más extenso, probablemente el diagnóstico más tardío de los tumores en los hombres, junto con la mayor frecuencia de tumores en regiones de mal pronóstico, tengan relación con el ensombrecimiento del pronóstico en este grupo de población. También existe una diferencia estadísticamente significativa entre la localización del tumor entre hombres y mujeres, siendo en áreas de peor pronóstico en el primer grupo.

Un $50 \%$ de la población a estudio resultó negativo en el estudio del ganglio centinela. El $25.74 \%$ de los casos fueron positivos. Curiosamente, se considera que la linfoscintigrafía (el estudio del ganglio centinela con radioisótopos) tiene una tasa de éxitos del $98 \%(6,13)$, sin embargo, en nuestro estudio, existen 15 pacientes en los que no se detectó ningún "punto caliente". Para conocer a qué se debe este alta tasa de fallidos, deben tenerse en cuenta las localizaciones de los melanomas, ya que no se presentó ninguno de ellos en el miembro inferior, y 12 de los 15 estaban localizados en el tronco la cara y el cuello, donde las líneas de Sappey se presentan superpuestas, lo que supone, según diversos estudios, un drenaje linfático impredecible o bilateral $(11,20,21)$. Sin embargo, entre los 78 pacientes, la localización más frecuente
Gráfico II.

Test of Equality over Strata

\begin{tabular}{lrrr} 
Test & Chi-Square & DF & $\begin{array}{r}\text { Pr > } \\
\text { Chi-Square }\end{array}$ \\
Log-Rank & 0.2033 & 1 & 0.6520 \\
Wilcoxon & 0.2219 & 1 & 0.6376 \\
-2Log(LR) & 0.1890 & 1 & 0.6638 \\
\hline
\end{tabular}

fue el miembro inferior (MI) (34.6\%), siendo la cara y cuello la localización menos frecuente de los melanomas $(11.53 \%)$.

En un seguimiento medio cercano a los dos años, se ha perdido el seguimiento de 13 pacientes y 7 de ellos han fallecido. De los restantes, el 81\% (47 de 58 casos) se encuentran en la actualidad libres de tumor.

Se considera que aproximadamente en todas las series existe un 3\% de falsos negativos (6). En nuestra serie se observan un $3.85 \%$ de falsos negativos a los dos años de seguimiento. No parece existir ninguna relación entre el tipo de tumor, la localización del tumor primario, ni el tiempo de demora entre la ampliación de márgenes y el ganglio centinela. Sin embargo, sí se trata de melanomas con espesor de Breslow mayor que la media, en los que tal vez habría que suponer que el ganglio negativo se trataba de un falso negativo.

Podemos comprobar cómo la anatomía patológica del ganglio centinela sí se corresponde con el pronóstico, puesto que la posibilidad ( hazard ratio) de desarrollar metástasis en caso de G.C. (+) es de 3.105 (IC: 1.020-9.452, p:0.0460).

Igualmente en nuestra serie el Breslow es un importante factor pronóstico. El Breslow medio de los pacientes libres de enfermedad es de $2.43 \mathrm{~mm}$, mientras que sube a $3.68 \mathrm{~mm}$ en aquellos que desarrollaron metástasis a lo largo del estudio. El hazard ratio de desarrollar metástasis para un espesor de Breslow mayor de $4 \mathrm{~mm}$ es de 15.340, p:0.0139. 
Aunque se ha recomendado que la ampliación de márgenes se realice en el mismo tiempo quirúrgico que el ganglio centinela puesto que los canales linfáticos pueden verse interrumpidos quirúrgicamente en la extirpación de la lesión primaria y pueden artefactar el resultado, lo cierto es que en nuestra casuística no se observa ninguna diferencia entre los pacientes con ampliación de márgenes previa y los pacientes con ampliación de márgenes en el mismo tiempo quirúrgico. Suponiendo que una de las causas de que el ganglio centinela fallido pudiese ser la ampliación previa (demora sí), comprobamos que el $20.34 \%$ de los ganglios con demora resultaron fallidos, al igual que el $16.6 \%$ de los ganglios sin demora, con lo cual, no observamos diferencias entre los grupos con ampliación de márgenes previa y sin ella en cuanto a la posibilidad de presentar ganglio centinela fallido.

Del mismo modo, para descartar que la ampliación de márgenes previa aumentara el riesgo de ganglio centinela positivo, comparamos ambos grupos para comprobar que, de los G.C. (+), el 75.0\% había tenido ampliación previa. El 76.9\% de los G.C. (-), también, por lo que no se observan diferencias entre ambos grupos.

Por último, quisimos comparar el pronóstico de los grupos con ampliación de márgenes previa y sin ella, observando que el $23.73 \%$ de los pacientes con ampliación presentaron metástasis en el tiempo de seguimiento, igual que el $22.22 \%$ de los pacientes sin ampliación previa.

\section{Conclusiones}

Nuestra serie corrobora que el espesor de Breslow y la anatomía patológica del ganglio centinela son factores pronósticos importantes en la evolución del melanoma maligno, aunque las conclusiones a las que podemos llegar son limitadas debido al tamaño de la muestra. Sería preciso un estudio más avanzado para comprobar si realmente existe un peor pronóstico en varones que en mujeres y si esto está relacionado con la localización del tumor y/o con el espesor de Breslow.

Con los datos obtenidos y presentados anteriormente, parece claro concluir que la ampliación de márgenes en un tiempo previo no influye en la detección del ganglio centinela ni ensombrece el pronóstico del melanoma, puesto que no se observan diferencias entre los grupos con demora y sin ella.

\section{Agradecimientos}

Quisiéramos agradecer de forma especial a la Unidad de Epidemiología del Hospital Doce de Octubre por su colaboración en la realización de este trabajo.

\section{Dirección del autor}

Dra. Eunate Martí Carrera

Hermanos Machado 5-7 T. 4- ${ }^{\circ}$ A

28660 Boadilla del Monte. Madrid

e-mail: eunatemarti@telefonica.net

\section{Bibliografía}

1. Morton DL, Wen D-R, Wong JH, et al. "Technical Details of Intraoperative Lymphatic Mapping for Early Stage Melanoma", Arch Surg, 1992; 127: 392

2. Balch CM, Wilkerson JA, Murad TM, et al. "The prognostic significance of ulceration of cutaneous melanoma", Cancer 1980; 45:3012.

3. Balch CM, Soong SJ, Murad TM, et al. "A multifactorial analysis of melanoma,II. Prognostic factors in patients with stage I (localized)melanoma". Surgery,1979; 86:343.

4. Gershenwald JE, Thompson W, Mansfield PF. "Multi-institutional melanoma lymphatic mapping experience: The prognostic value of sentinela lymph node status in 612 stage I or II patients", J Clin Oncol, 1999; 17:976

5. McKie RM, Byrne D, Lyngman MK. "Prognostic evaluation os sentinel node biopsy", Melanoma Res, 1997; 7:102

6. Ling-Xi L., Scolyer R.A., Ka V.S., et al. "Pathologic review of Negative Sentinel Lymph Nodes in Melanoma Patients With Regional Recurrence", Am J Surg Pathol. 2003; 27:1197.

7. Goldstein BG, Goldstein AO. "Diagnosis and management of malignant melanoma", Am Fam Phys, 2001, 63:1359

8. Mariani G, Erba P, Manca G, et al. "Radioguided sentinel lymph node biopsy in patients with malignant cutaneous melanoma: the nuclear medicine contribution", J Surg Oncol, 2 004; 85(3): 141.

9. Meyer CM, Lecklitner ML, Logic JR, et al. "Technetium-99m sulfur-coloid cutaneous lymphoscintigraphy in the management of truncal melanoma", Radiology, 1979; 131:205.

10. Rees WV, Robinson DS, Holmes EC, et al. "Altered lymphatic drainage following lymphadenectomy", Cancer, 1980;45:3045.

11. Berman CG, Norman J, Cruse CW, et al. "Lymphoscintigraphy in malignant melanoma", Ann Plast Surg, 1992;28:29.

12. Leong SP, Ghazarian DM, McCready DR, et al. "Previous wide local excision of primary melanoma is not a contraindication for sentinel lymph node biopsy of the trunk and extremity", J Surg Oncol, 2003; 82: 143.

13. Leong SP, Thelmo MC, Kim RP, et al. "Delayed harvesting of sentinel lymph nodes after previous wide local excision of extremity melanoma", Ann Surg Oncol, 2003; 10:196

14. McCready DR, Ghazarian DM, Hershkop MS et al. "Sentinel lymph node biopsy after wide local excision for melanoma", Can J Surg, 2001; 44:432.

15. Balch, C.M ; Buzaid, A.C.; Soong, S.J.; et al. "Final Version of the American Joint Committee on Cancer Staging System for Cutaneous Melanoma", J Clin Oncol 2001, 19:3635.

16. Gershenwald JE, Colome MI, Lee JE. "Patterns of Recurrence following a negative sentienl lymph node biopsy in 243 patients with stage I or II melanoma", J Clin Oncol, 1998; 15:2253

17. Gibbs JF, Huang PP, Zhang PJ, et al. "Accuracy of pathologic techniques for the diagnosis od metastatic melanoma in sentinel lymph node", Ann Surg Oncol,1999;6:699

18. Yu LL, Flotte TJ, Tanabe KK, et al. "Detection of microscopic melanoma metastases in sentienl lymph nodes", Cancer,1999; 86:617

19. Bostick PJ, Morton DL, Turner RR. "Prognostic significance of occultt metastases detected by sentienl lymphadenectomy and reverse transcripatse-polymerase chain reaction in early stage melanoma patients", J Clin Oncol, 1999; 17:3238

20. Eberbach MA, Wahl RL, Argenta LC, et al. "Utility of lymphoscintigraphy in directing surgical therapy for melanomas of the head, neck and upper thorax", Surgery, 1978; 84:626.

21. Tulley P., Butler, P.E. "Sentinel Lymph Node Biopsy for Malignant Melanoma", Plast Reconstr Surg, 2001; 108 (7):2169 


\section{Comentario al trabajo uEl ganglio centinela en eI melanoma: nuestra experiencian}

\author{
Dr. Antonio Bazán \\ Director del Servicio de Cirugía \\ Plástica, Estética y Reparadora \\ Clínica Universitaria de Navarra \\ Pamplona (España)
}

Antes que nada desearía felicitar a los autores por el trabajo realizado, tanto en su planteamiento de base, como en su ejecución metodológica y estadística. La importancia del ganglio centinela en el diagnóstico, tratamiento y pronóstico del melanoma es, en la actualidad, indudable. En este contexto, la intención de los autores de analizar la importancia real del factor "tiempo de demora" entre la ampliación de márgenes del melanoma primario y la determinación del ganglio centinela en su serie resulta especialmente interesante, puesto que es éste un tema de amplia controversia.

Únicamente, me permito hacer unas pequeñas consideraciones que creo pueden ser de interés para una visión contrastada del tema.

Está bien establecido que la mayor indicación para le realización del ganglio centinela es, como bien apuntan los autores, el paciente con Breslow $>1 \mathrm{~mm}$ y sin adenopatías palpables (estadíos I y II de la AJCC). Sin embargo, no parece muy claro que esta técnica tenga un especial beneficio en pacientes con Breslow superior a $4 \mathrm{~mm}$, dada la más que probable extensión, cuando menos, regional. De hecho, en los datos que se muestran en el estudio así parece cororroborarse (pacientes que desarrollaron metástasis en menos de un año tras falso negativo). Este es un grupo de pacientes de melanoma con pronóstico muy heterogéneo, ya que, según estudios $(1,2)$, hasta el $50 \%$ de ellos tienen enfermedad ganglionar oculta ya en el diagnóstico, por lo que, teóricamente y en términos de control local, podrían beneficiarse de una linfadenectomía terapéutica. Pero, en cualquier caso, esto no afecta al objetivo del trabajo, es decir al análisis de la correlación entre el momento de la ampliación y la determinación del GC.

Otro punto a discusión sería el de la eficacia del ganglio centinela en pacientes a los que se les ha realizado una ampliación de márgenes previa extensa, que es dudosa o, al menos desconocida, porque se ha alterado el patrón anatómico normal de drenaje linfático, lo cual puede llevar a una incorrecta identificación del GC. Sin embargo, aunque todo esto es anató- micamente lógico, algunos centros han publicado recientemente algunas series limitadas, sugiriendo que tras una ampliación de márgenes de hasta $1 \mathrm{~cm}$, la técnica de GC parece ser fiable (3) (en el trabajo los autores plantean unos márgenes consensuados de 1 ó $2 \mathrm{~cm}$, según el Breslow).

Permítaseme una pequeña nota acerca de la ampliación de márgenes de la lesión primaria: estoy completamente de acuerdo en que para el cierre de la misma, si se va a realizar una determinación de GC diferida no es apropiada la utilización de colgajos o plastias locales, siendo el cierre directo la técnica de elección; cosa distinta sería en un caso simultáneo, sobre todo si se trata de la cara especialmente. No obstante, no parece muy razonable el cierre por segunda intención máxime cuando el tiempo medio hasta la determinación del GC es de 5,3 meses. Evidentemente es éste un factor no dependiente del equipo quirúrgico receptor del caso para GC pero sí puede hacernos reflexionar sobre la importancia de la colaboración interdisciplinar ágil e integradora entre los distintos actores implicados en el proceso terapéutico, máxime cuando de un proceso oncológico se trata.

Como hemos comentado, desde un punto de vista de lógica anatómica y clínica, la determinación del GC debería aportar los mayores beneficios diagnósticos y pronósticos si se realizara simultáneamente o, al menos, en el menor tiempo posible la ampliación de márgenes del melanoma primario En cualquier caso, los resultados que muestran los autores tras el completo análisis estadístico aportan unos resultados muy interesantes, demostrando en su serie que la ampliación de márgenes en un tiempo previo no influye en la detección del ganglio centinela.

Por último quisiera nuevamente felicitar a los autores, no sólo por el espléndido trabajo sino, también, y sobre todo, por su implicación y esfuerzo en el tratamiento interdisciplinar de esta entidad clínica en la cual, sin lugar a dudas, la Cirugía Plástica tiene mucho que decir y, sobre todo, que aportar, con su visión quirúrgica global e integral del proceso terapéutico. 


\section{Bibliografía}

1. Balch, CM, Soong S, Roos MI, Urist MM, Karakousis CP, Temple WJ, Mihm MC, Barnhill RL, Jewell WR, Wanebo HJ, Harrison R: "Long-Term Results of a Multi-Institutional Randomized Trial Comparing Prognostic Factors and Surgical Results for Intermediate Thikness Melanomas (1.0 to 4.0)". Intergroup Melanoma Surgical Trial. Ann Surg Oncol 2000; 7(2): 87.
2. Gershenwald JE, Mansfield PF, Lee JE, Ross MI: "Role for Lymphatic Mapping and Sentinel Node Biopsy in Patients with Thick ( $>$ or $=4 \mathrm{~mm}$ ) Primary Melanoma". Ann Surg Oncol 2000; 7: 226

3. Rousseau DJ, Ross MI, Lee JE, Mansfield PF, Gershenwald JE. "Accuracy of sentinel node biopsy after wide excision in melanoma patients. Proceedings of SSO 2003.

\section{Respuesta al comentario del Dr. A. Bazán}

\section{Dra. E. Martí Carrera}

En primer lugar quería agradecer sinceramente al Dr. Bazán su comentario y el interés mostrado en nuestro trabajo. Si bien estamos completamente de acuerdo con sus matizaciones, creemos que, dado que hablamos de una técnica relativamente joven, existen aún muchos puntos de controversia. Además, al tratarse de un procedimiento que precisa la actuación de diversos especialistas, es inevitable que, en hospitales con gran carga asistencial, exista cierta demora en la actuación que, entre todos, debemos esforzarnos en corregir. 